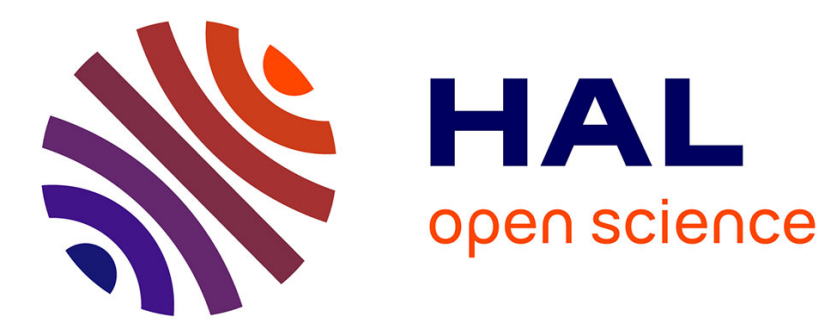

\title{
Robust Surface Reconstruction via Triple Sparsity
}

Hicham Badri, Hussein Yahia, Driss Aboutajdine

\section{To cite this version:}

Hicham Badri, Hussein Yahia, Driss Aboutajdine. Robust Surface Reconstruction via Triple Sparsity.

CVPR 2014, PAMITC, Jun 2014, Columbus, Ohio, United States. hal-00951627

\section{HAL Id: hal-00951627 \\ https://hal.inria.fr/hal-00951627}

Submitted on 4 Apr 2014

HAL is a multi-disciplinary open access archive for the deposit and dissemination of scientific research documents, whether they are published or not. The documents may come from teaching and research institutions in France or abroad, or from public or private research centers.
L'archive ouverte pluridisciplinaire HAL, est destinée au dépôt et à la diffusion de documents scientifiques de niveau recherche, publiés ou non, émanant des établissements d'enseignement et de recherche français ou étrangers, des laboratoires publics ou privés. 


\section{Robust Surface Reconstruction via Triple Sparsity}

\author{
Hicham Badri \\ INRIA, Geostat team \\ 33400 Talence, France \\ hicham.badrieinria.fr
}

\author{
Hussein Yahia \\ INRIA, Geostat team \\ 33400 Talence, France \\ hussein.yahia@inria.fr
}

\author{
Driss Aboutajdine \\ LRIT - CNRST (URAC29) \\ 1014 RP Rabat, Morocco \\ aboutajeieee.org
}

\begin{abstract}
Reconstructing a surface/image from corrupted gradient fields is a crucial step in many imaging applications where a gradient field is subject to both noise and unlocalized outliers, resulting typically in a non-integrable field. We present in this paper a new optimization method for robust surface reconstruction. The proposed formulation is based on a triple sparsity prior : a sparse prior on the residual gradient field and a double sparse prior on the surface gradients. We develop an efficient alternate minimization strategy to solve the proposed optimization problem. The method is able to recover a good quality surface from severely corrupted gradients thanks to its ability to handle both noise and outliers. We demonstrate the performance of the proposed method on synthetic and real data. Experiments show that the proposed solution outperforms some existing methods in the three possible cases : noise only, outliers only and mixed noise/outliers.
\end{abstract}

\section{Introduction}

Reconstruction from corrupted gradient fields is a task of primary importance in several imaging applications. For instance, recovering the surface shape from captured images using Photometric Stereo (PS) [17] and Shape from Shading (SFS) [8] requires robust surface reconstruction tools to integrate surface normal vectors. Many computational photography applications such as HDR compression [5], image editing [12], stitching [10], super-resolution [16], manipulate image gradients and reconstruct a new image from the resulting gradient field. Integration is also used to recover an image from its incomplete Fourier measurements after estimating the corresponding gradients using Compressed Sensing methods [11]. In all the applications above, the resulting gradient field is non-integrable either due to errors in measurements including noise and/or outliers, or the corresponding gradient field is directly modified by mixing multiple gradients of different images, or simply applying linear/nonlinear functions. This paper proposes a new opti- mization method for robust surface reconstruction from corrupted gradient fields. Unlike previous optimization formulations [15, 14, 4, 2, 7], we consider a triple sparsity prior : a double sparse prior, on the gradient residual and the surface gradients, aims at efficiently handling gradient field outliers. A third sparse prior improves reconstruction quality in the case of gradient noise. The contributions are as follows :

- We present a new optimization method for robust reconstruction of sparse gradient signals from corrupted gradient fields, handling both noise and outliers.

- We propose an efficient alternate minimization strategy to solve the proposed problem.

- We demonstrate the performance of the proposed framework on synthetic and real data and compare it to some existing reconstruction methods.

\section{Related Work}

Enforcing integrability can be traced back to the work of Chellappa et al. [15, 6] for problems such as Shape from Shading. Poisson reconstruction [6] is probably the most popular approach to integration. It consists in solving the Poisson equation that is derived from a straightforward least-squares fit. It is well known that least squares solutions are not robust to outliers [14]. What happens in the problem of integration is that, when using a least squares solution, the errors are propagated and can result in an unnatural surface/image even if only few gradient points are corrupted as can be seen in Figure 11. The FrankotChellappa method [15] performs a projection of the nonintegrable gradient field in the Fourier basis. The technique was extended to non-orthogonal set of basis functions such as shapelets [9]. Petrovic et al. [13] propose a loopy belief propagation integration method when the gradient field is corrupted with a Gaussian noise. Agrawal et al. propose in [2] a more general framework to extend the Poisson equation. Another method by Agrawal et al. consists in correcting the gradient field with an algebraic method [1]. The method produces impressive results when the gradient field 
is corrupted with outliers only, but unfortunately seems less satisfactory in the presence of noise. The methods in [7] use spectral and Tikhonov regularizations to improve least squares fitting. The techniques above can either work relatively well in the case of noise only or outliers only, but not when both noise and outliers are mixed, which is usually the case of real world data. More sophisticated methods that better handle outliers are based on $l_{1}$-minimization. The method in [14] addresses the problem as a residual gradient error correction considering the $l_{1}$-norm. Another method presented in [4] searches for a best fit in the $l_{1}$-norm sense ; $l_{1}$-based methods [14, 4] can handle the three cases but these methods are unable to recover a good quality surface when the gradient field is severely corrupted.

The solution proposed in this paper considers a triple sparsity prior. A double sparsity prior, on the residual gradient and the surface gradients, is considered to better handle gradient outliers. Another sparse prior on the surface is considered to better deal with noisy gradients and produce a smooth surface. The $l_{1}$-norm is a popular choice to induce sparsity. However, it does not model well gradient outliers. In fact, using the $l_{1}$-norm comes to consider a Laplacian distribution $\left(p(x) \propto e^{-\tau|x|}\right)$, which is not enough heavily tailed to properly model the strong sparsity of the residual gradient field in the case of outliers. We consider the $l_{p<1^{-}}$ norm (Hyper-Laplacian distribution) to model this residual gradient sparsity. The second and third sparse priors are presented in a regularization form. They consist in additional information known about the signal to improve the reconstruction quality. As surfaces are smooth, the sparse natural priors are considered in the gradient domain. We show that this setup can significantly improve reconstruction quality compared to previous methods, especially in challenging mixed noise/outliers cases.

\section{Problem Statement}

Let $S(x, y)$ be the desired surface to recover. We denote by $s$ its vectorized form of length $n$. Let $\vec{v}=(p, q)$ denote the given non-integrable gradient field and $\nabla s=\left(s_{x}, s_{y}\right)$ the true gradient of $S$. Integration consists in recovering $s$ from $\vec{v}$, commonly formulated in the following form :

$$
\hat{s}=\underset{s}{\operatorname{argmin}} \phi_{r}(\nabla s-\vec{v}) .
$$

where $\phi_{r}$ models the distribution of the residual gradient error. $\phi_{r}=\|.\|_{2}^{2}[15]$ and $\phi_{r}=\|.\|_{1}[4]$ are special cases.

The proposed formulation is substantially different : it consists in a double sparsity prior using regularization to efficiently handle outliers, and a third sparsity prior for noiseaware reconstruction :

$\underset{s, s^{\prime}}{\operatorname{argmin}} \underbrace{\left\|\nabla s^{\prime}-\vec{v}\right\|_{p_{1}}^{p_{1}}+\lambda_{1}\left\|\nabla s^{\prime}\right\|_{p_{2}}^{p_{2}}}_{\text {robust recovery }}+\underbrace{\frac{\gamma}{2}\left\|s-s^{\prime}\right\|_{2}^{2}+\lambda_{2}\|\nabla s\|_{p_{3}}^{p_{3}}}_{\text {smoothing }}$,

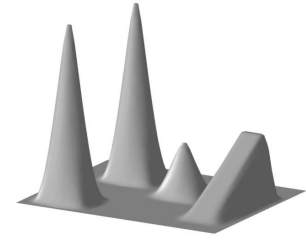

(a) Ground Truth

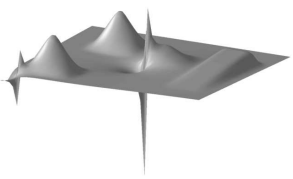

(c) Poisson reconstruction

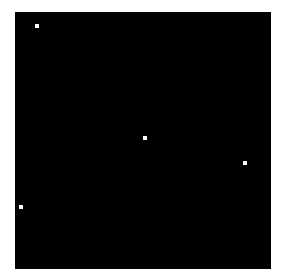

(b) Gradient outliers location

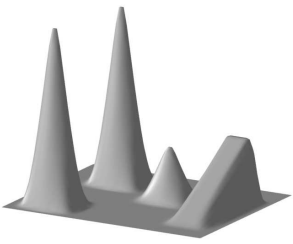

(d) Proposed method

Figure 1: Least squares fitting example in the case of outliers. As can be seen, Poisson reconstruction (c) produces a deformed surface even in the presence of very few outliers. The proposed method efficiently handles outliers and produces a high quality surface (d).

where $\lambda_{1}, \lambda_{2}$ and $\gamma$ are positive regularization terms, $s^{\prime}$ is an intermediate surface and $p_{1}, p_{2}, p_{3}<1$. Problem (2) is highly non-convex, we use a half-quadratic approach to estimate a solution by introducing additional variables $v_{1}, v_{2}, v_{3}$ :

$$
\begin{gathered}
\underset{s, s^{\prime}, v_{1}, v_{2}, v_{3}}{\operatorname{argmin}}\left\|v_{1}\right\|_{p_{1}}^{p_{1}}+\frac{\beta_{1}}{2}\left\|\left(\nabla s^{\prime}-\vec{v}\right)-v_{1}\right\|_{2}^{2} \\
\quad+\lambda_{1}\left\{\left\|v_{2}\right\|_{p_{2}}^{p_{2}}+\frac{\beta_{2}}{2}\left\|\nabla s^{\prime}-v_{2}\right\|_{2}^{2}\right\} \\
+\frac{\gamma}{2}\left\|s-s^{\prime}\right\|_{2}^{2}+\lambda_{2}\left\{\left\|v_{3}\right\|_{p_{3}}^{p_{3}}+\frac{\beta_{3}}{2}\left\|\nabla s-v_{3}\right\|_{2}^{2}\right\},
\end{gathered}
$$

where $\beta_{i}, i=1,2,3$ are new positive regularization terms that are increased by multiplying $\beta_{i}$ with $\kappa_{i}>1$ at each iteration. The optimization problem is split into subproblems $\left(p_{i}\right)$ solved iteratively :

$$
\begin{gathered}
\left(p_{1}\right): v_{1}^{(k+1)} \leftarrow \underset{v_{1}}{\operatorname{argmin}}\left\|v_{1}\right\|_{p_{1}}^{p_{1}}+\frac{\beta_{1}}{2}\left\|\left(\nabla s^{\prime(k)}-\vec{v}\right)-v_{1}\right\|_{2}^{2} \\
\left(p_{2}\right): v_{2}^{(k+1)} \leftarrow \underset{v_{2}}{\operatorname{argmin}}\left\|v_{2}\right\|_{p_{2}}^{p_{2}}+\frac{\beta_{2}}{2}\left\|\nabla s^{(k)}-v_{2}\right\|_{2}^{2} \\
\left(p_{3}\right): s^{(k+1)} \leftarrow \underset{s^{\prime}}{\operatorname{argmin}} \gamma\left\|s^{\prime}-s^{(k)}\right\|_{2}^{2}+\beta_{1} \|\left(\nabla s^{\prime}-\left(\vec{v}+v_{1}^{(k+1)}\right) \|_{2}^{2}\right. \\
+\lambda_{1} \beta_{2}\left\|\nabla s^{\prime}-v_{2}^{(k+1)}\right\|_{2}^{2}, \nabla s^{(k)} \leftarrow \nabla s^{\prime(k+1)} \\
\left(p_{4}\right): v_{3}^{(k+1)} \leftarrow \underset{v_{3}}{\operatorname{argmin}}\left\|v_{3}\right\|_{p_{3}}^{p_{3}}+\frac{\beta_{3}}{2}\left\|\nabla s^{(k)}-v_{3}\right\|_{2}^{2} \\
\left(p_{5}\right): s^{(k+1)} \leftarrow \underset{s}{\operatorname{argmin} \gamma \|} \gamma-s^{\prime(k)}\left\|_{2}^{2}+\lambda_{2} \beta_{3}\right\| \nabla s-v_{3}^{(k+1)} \|_{2}^{2} \\
\nabla s^{(k+1)} \leftarrow \nabla s^{(k+1)}, \beta_{1} \leftarrow \kappa_{1} \beta_{1}, \beta_{2} \leftarrow \kappa_{2} \beta_{2}, \beta_{3} \leftarrow \kappa_{3} \beta_{3}
\end{gathered}
$$

3.1. Solving problems $\left(p_{1}\right),\left(p_{2}\right)$ and $\left(p_{4}\right)$

Problems $\left(p_{1}\right),\left(p_{2}\right)$ and $\left(p_{4}\right)$ are in the proximal form :

$$
\underset{v}{\operatorname{argmin}}\|v\|_{p}^{p}+\frac{\beta}{2}\|x-v\|_{2}^{2}
$$


the solution is given via generalized soft-thresholding [3] :

$$
v=\operatorname{shrink}_{l_{p}}(x, \beta)=\max \left\{0,|x|-\frac{|x|^{p-1}}{\beta}\right\} \frac{x}{|x|} .
$$

The special case of $p=0$ consists in hard-thresholding [18]

$$
v=\operatorname{shrink}_{l_{0}}(x, \beta)= \begin{cases}0 & |x|^{2} \leq \frac{2}{\beta} \\ x & \text { otherwise. }\end{cases}
$$

Note however that $v$ in our case is a 2-components vector. We adopt an anisotropic approach which consists in applying the thresholding on each vector field component separately. Thus, the solutions to problems $\left(p_{j}\right), j=1,2$ are

$$
v_{j}^{(k+1)}=\left\{\begin{array}{l}
v_{j, x}^{(k+1)}(i)=\operatorname{shrink}_{l_{p}}\left(\nabla_{x} s^{(k)}(i)-v_{x}(i), \beta_{j}\right) \\
v_{j, y}^{(k+1)}(i)=\operatorname{shrink}_{l_{p}}\left(\nabla_{y} s^{(k)}(i)-v_{y}(i), \beta_{j}\right) \\
i=1, \ldots, n,
\end{array}\right.
$$

and the solution to problem $\left(p_{3}\right)$ is given as follows :

$$
v_{3}^{(k+1)}=\left\{\begin{array}{l}
v_{3, x}^{(k+1)}(i)=\operatorname{shrink}_{l_{p}}\left(\nabla_{x} s^{(k)}(i), \beta_{3}\right) \\
v_{3, y}^{(k+1)}(i)=\operatorname{shrink}_{l_{p}}\left(\nabla_{y} s^{(k)}(i), \beta_{3}\right) \\
i=1, \ldots, n .
\end{array}\right.
$$

\subsection{Solving problems $\left(p_{3}\right)$ and $\left(p_{5}\right)$}

Problem $\left(p_{5}\right)$ is quadratic and easy to solve via EulerLagrange equation. The solution can be computed either by solving a linear system such that $\nabla x \approx\left(D_{x} x, D_{y} x\right)$, where $D_{x}$ and $D_{y}$ are differential operators in the matrix form, or performing a deconvolution using the Fourier transform $\mathcal{F}$ such that $\nabla x \approx\left(x \star\left[\begin{array}{ll}1 & -1\end{array}\right]^{T}, x \star\left[\begin{array}{ll}1 & -1\end{array}\right]\right)$, where $\star$ is the convolution operator. Considering periodic boundary conditions, we choose the Fourier transform method that gives the following solution :

$$
s^{(k+1)}=\mathcal{F}^{-1}\left(\frac{\mathcal{F}\left(\gamma s^{(k)}-\lambda_{2} \beta_{3} \operatorname{div}\left(v_{3}^{(k+1)}\right)\right)}{\gamma-\lambda_{2} \beta_{3} \operatorname{lap}}\right) .
$$

where div is the discrete divergence operator and lap is the Fourier transform of the discrete Laplacian filter. Problem $\left(p_{3}\right)$ is similar. By applying the Euler-Lagrange equation and considering the Fourier method, the solution to problem $\left(p_{3}\right)$ is given as follows :

$$
\begin{aligned}
& s^{\prime(k+1)}=\mathcal{F}^{-1}\left(\frac{\mathcal{F}\left(\gamma s^{(k)}-\operatorname{div}(u)\right)}{\gamma-\left(\beta_{1}+\lambda \beta_{2}\right) \operatorname{lap}}\right) \\
& u=\beta_{1}\left(\vec{v}+v_{1}^{(k+1)}\right)+\lambda \beta_{2} v_{2}^{(k+1)} .
\end{aligned}
$$

\subsection{Justification}

To show why and how the proposed approach improves the quality of the reconstruction, we propose to study separately the robust reconstruction step which consists in solving problems $\left(p_{1}\right),\left(p_{2}\right)$ and $\left(p_{3}\right)$ in the case of strong outliers, and then show why problems $\left(p_{4}\right)$ and $\left(p_{5}\right)$ are important in the case of noisy gradients.

\subsubsection{Why Double Sparsity for Outliers?}

The proposed formulation (2) is composed of two parts : a double sparsity part for robust recovery and a sparsity prior for smoothing. We consider in this section only the robust recovery part $\left(\gamma=0\right.$ and $\left.\lambda_{2}=0\right)$ to see how the proposed recovery formulation improves reconstruction in the case of strong outliers. The robust reconstruction step consists in solving the following optimization problem :

$$
\underset{s^{\prime}}{\operatorname{argmin}}\left\|\nabla s^{\prime}-\vec{v}\right\|_{p_{1}}^{p_{1}}+\lambda_{1}\left\|\nabla s^{\prime}\right\|_{p_{2}}^{p_{2}} .
$$

Outliers consist in sparse errors with strong magnitude (due for instance to depth discontinuities and shadows). A least squares fit $\left(p_{1}=2\right.$ and $\left.\lambda_{1}=0\right)$ tends to propagate errors and results in a corrupted surface as it was shown in Figure 1. The reason why this happens is that, when using the $l_{2}$ norm, the distribution of the residual gradient error $\nabla s^{\prime}-\vec{v}$ is modeled using a Gaussian which is not appropriate when the gradient is corrupted with outliers only. Instead, the model should take into account the sparsity of the residual gradient, which comes to the cost of using the $l_{p \leq 1}$ norm. However, the $l_{1}$-norm does not model well either the strong sparsity of the residual gradient, which makes the recovery of the surface only possible when the number of outliers is relatively low. Thus, our choice for the $l_{p<1}$-norm which models better the heavy-tailed distribution of the gradient errors in the case of outliers. Note that, using the $l_{p<1}$ norm alone, the performance is rather limited. In fact, using the $l_{p}$-norm on the residual gradients is a maximum likelihood (MP) estimation which can be improved with a MAP estimation instead. The MAP estimation in our case consists in regularizing the MP estimation with a natural prior. The natural prior that we choose is the smoothness of the surface itself, hence the use of the $l_{p}$-norm on the gradient of $s^{\prime}$ too.

To properly evaluate the importance of the proposed double sparsity model, we run reconstruction experiments on the Shepp-Logan phantom instance and study the case of exact recovery of the image from corrupted gradients with outliers. We use this image because it is a standard benchmark instance for exact recovery in Compressed Sensing applications. Note that here, we reconstruct the image from corrupted gradients with outliers and not incomplete Fourier measurements as the image instance is usually used for. Outliers are generated as sparse random errors with a strong magnitude. We study the case of $l_{2}$, diffusion [2], $l_{1}$, $l_{p}(p=0.1)$ and the proposed double $l_{p}(p=0.1$ for both $p_{1}$ and $p_{2}$ ) model and present the results in Figure 2. As can be seen, the proposed method is able to recover exactly the original image even in the presence of a high level of outliers. 


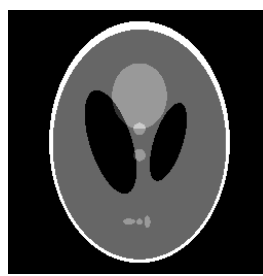

(a) Ground Truth

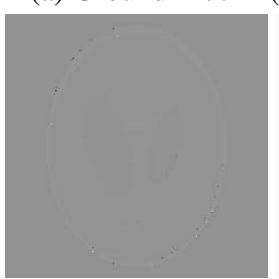

(d) $l_{1}[14$

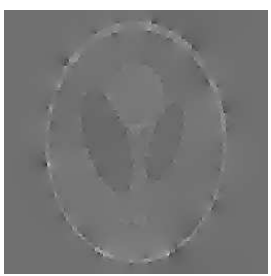

(b) Least Squares [15

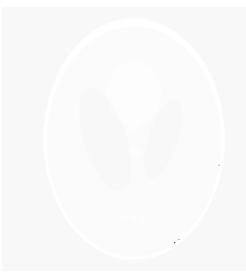

(e) $l_{p<1}$ fit (f) Double $l_{p<1}$

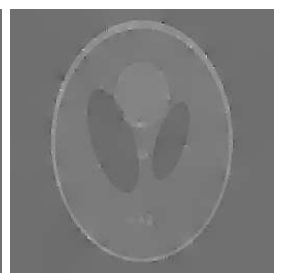

(c) Diffusion [2]

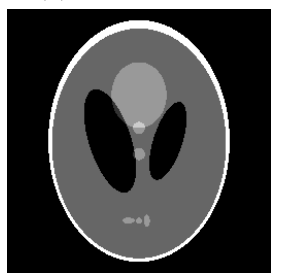

Figure 2: Reconstruction quality comparison between the proposed double sparsity model and some other methods. Exact recovery is possible with the proposed method even in the case of strong outliers (more than $12 \%$ of the gradient points were corrupted with random sparse high magnitude errors. The magnitude of the outliers is 10 times the maximum of the gradient norm $\max \left(\sqrt{\nabla_{x} s^{2}+\nabla_{y} s^{2}}\right)$.

\subsection{Why a Third Sparsity for Noise?}

We saw in the previous section how the double sparsity prior improves the quality of recovery in the case of outliers. Note however, that when solving problem (12), which consists in solving sub-problems $\left(p_{1}\right),\left(p_{2}\right)$ and $\left(p_{3}\right)$, there is no step to smooth the surface in the case of noise. Thus, using the formulation (12) for the mixed noise/outliers case can successfully correct outliers but cannot denoise the surface. Hence, we introduce a denoising step which consists in using another sparse gradient prior as follows :

$$
\underset{s}{\operatorname{argmin}} \frac{\gamma}{2}\left\|s-s^{\prime}\right\|_{2}^{2}+\lambda_{2}\|\nabla s\|_{p_{3}}^{p_{3}}
$$

When problems (12) and (13) are combined, it gives problem (2) which is solved iteratively by correcting the outliers (problems $\left(p_{1}\right),\left(p_{2}\right)$ and $\left(p_{3}\right)$ ) followed by a denoising step (problems $\left(p_{4}\right),\left(p_{5}\right)$ ). To show the importance of this third sparsity model compared to the previous double sparsity model, we run reconstruction experiments on the SheppLogan phantom instance and study the case of near exact recovery of the image from corrupted gradients with noise and outliers. We generate a random Gaussian noise and the same amount of outliers as the previous section. Results are presented in Figure 3 As can be seen, improved recovery is obtained with the triple sparsity model. The double sparsity model corrects outliers but does not get rid of the noise.

Due to the high non-convexity of the proposed optimiza-

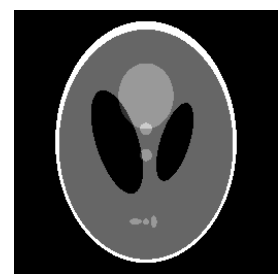

(a) Ground Truth

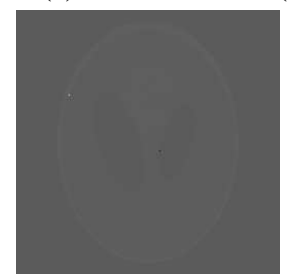

(d) $l_{p<1}$ fit

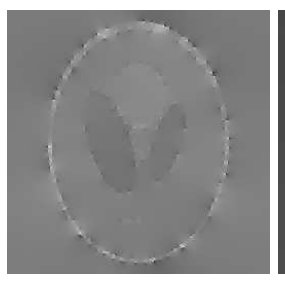

(b) Least Squares [15]

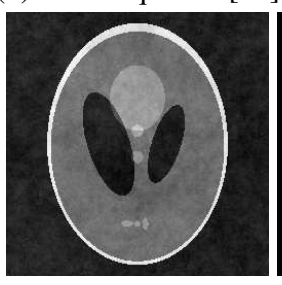

(e) Double $l_{p<1}$

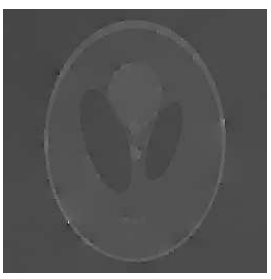

(c) Diffusion [2]

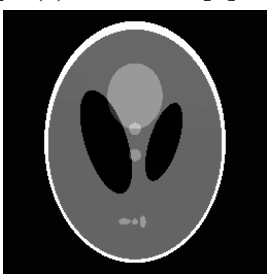

(f) Triple $l_{p<1}$
Figure 3: Reconstruction quality comparison in the case of both noise and outliers. The double sparsity model corrects outliers but does not denoise the output. The third sparsity prior permits to denoise the instance while correcting outliers resulting in a near-exact recovery $(\sigma=7 \%$ of the maximum intensity value, with the same outliers level as 2 .

tion problem, the half-quadratic solver cannot reach a global minimum. However, experiments show that the method converges to a local minimum after a certain number of iterations. The solver starts with a trivial solution (Poisson reconstruction) and iteratively corrects the vector field. If the gradients are error-free, the trivial solution is the true surface, which leads to a zero residual gradient error.

\section{Results}

To evaluate the proposed solution, we run multiple experiments including synthetic and real data. Similar to previous work, we first compare the method on the Ramp Peaks dataset which is a standard benchmark surface [2, 14, 4]. We use the same parameters so the reader can compare the results with other methods that can be found in the papers just cited. The second experiment consists in Photometric Stereo on the synthetic Mozart and Vase datasets used in [14, 2]. The third experiment consists in Photometric Stereo on real noisy images.

\subsection{Surface Reconstruction}

We corrupt a gradient field and try to reconstruct the surface from the resulting non-integrable field. We use the Ramp Peaks synthetic dataset considering the three cases. The MSE is reported in Table 1 .

Noise only : We add Gaussian noise to the ground truth gradient field and try to recover it. We take the same parameters as the previous work $\sigma=10 \%$ of the maxi- 


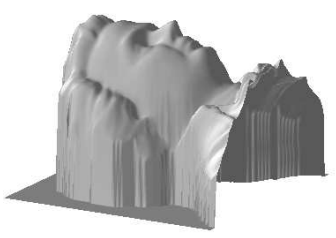

(a) Ground Truth

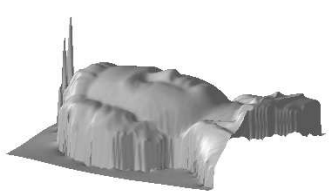

(d) M-estimator [2]

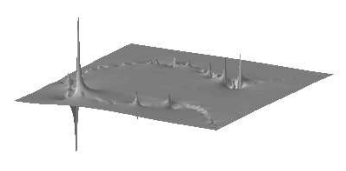

(b) Least Squares [15]

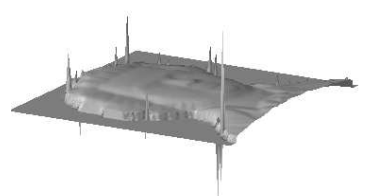

(e) $l_{1}$-minimization [14]

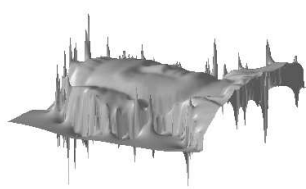

(c) Diffusion [2]

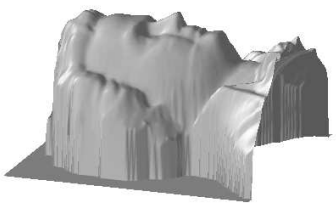

(f) Triple Sparsity

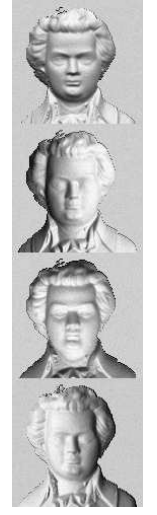

(g) Noisy input images

Figure 4: Photometric stereo on noisy Mozart dataset ( $\sigma=3 \%$ of the maximum intensity). The proposed method is able to recover a high quality surface from noisy images (parameters : $\lambda_{1}=0.25, \lambda_{2}=8 \times 10^{-5}, \gamma=10^{-4}, p_{1}=p_{2}=p_{3}=0.1$ ).

mum gradient value). Results are presented in Figure 6 (for $\lambda_{1}=0.05, \lambda_{2}=0.001, \gamma=0.4, p_{1}=p_{2}=p_{3}=0.5$ ). The sparse gradient prior is able to reduce the amount of the gradient noise and produce a smooth surface.

Outliers only : We add salt and pepper noise with a range five times that of the original gradient field which corresponds to outliers [14] (10\% of the gradient points are corrupted). Results are presented in Figure 7 (for $\lambda_{1}=0.4$, $\left.\gamma=10^{-5}, p_{1}=0.5, p_{2}=0.95\right)$. The double sparsity prior permits to correct more outliers than the $l_{1}$-minimization method thanks to the use of the $l_{p<1}$-norm and the sparse gradient prior, resulting in a near-exact recovery of the surface.

Mixed outliers/noise : We mix this time both noise and outliers. We consider $\sigma=7 \%$ of the maximum gradient value and corrupt $7 \%$ of the gradient points with outliers as suggested in [14]. Results are presented in Figure 8)(for $\lambda_{1}=0.33, \lambda_{2}=0.1, \gamma=0.01, p_{1}=0.5, p_{2}=p_{3}=0.2$ ). As can be seen, the proposed solution corrects outliers and produces a good quality smooth surface even in the presence of strong gradient noise and outliers.

\subsection{Photometric Stereo}

We evaluate the robustness of the proposed approach on calibrated photometric stereo experiments using synthetic and real sequences. Photometric stereo is a well known reconstruction method based on the powerful shading cue. Given a squence of three or more images of a 3D scene taken from the same viewpoint and under varying illumination, the method aims at reconstructing the 3D scene based on the intensity variation in each pixel. First, the surface normals are estimated, then integrated to produce a detailed estimate of the surface. The method however can fail due to the presence of shadows and noise, which introduce errors

\begin{tabular}{|c|c|c|}
\cline { 2 - 3 } \multicolumn{5}{c|}{} & Method & MSE \\
\hline \multirow{5}{*}{ Noise only } & Least Squares & 0.0432 \\
\cline { 2 - 3 } & Diffusion & 0.0519 \\
\cline { 2 - 3 } & M-estimator & 0.0482 \\
\cline { 2 - 3 } & $l_{1}$ & 0.0469 \\
\cline { 2 - 3 } & Triple Sparsity & $\mathbf{0 . 0 0 8}$ \\
\hline \multirow{5}{*}{ Outliers only } & Least Squares & 0.1437 \\
\cline { 2 - 3 } & Diffusion & 0.1171 \\
\cline { 2 - 3 } & M-estimator & 0.1314 \\
\cline { 2 - 3 } & $l_{1}$ & 0.1261 \\
\cline { 2 - 3 } & Triple Sparsity & $\mathbf{0 . 0 0 0 1}$ \\
\hline \multirow{5}{*}{ Mixed noise/outliers } & Least Squares & 0.1494 \\
\cline { 2 - 3 } & Diffusion & 0.0949 \\
\cline { 2 - 3 } & M-estimator & 0.1146 \\
\cline { 2 - 3 } & $l_{1}$ & 0.1016 \\
\cline { 2 - 3 } & Triple Sparsity & $\mathbf{0 . 0 2 1 2}$ \\
\hline
\end{tabular}

Table 1: Normalized MSE of the reconstructed surfaces on the Ramp Peaks dataset.

in the estimation of the surface normals, hence errors in the estimated gradient field. In this case, the corresponding gradient field is corrupted with both noise and outliers. A robust integration method is required to handle strong gradient field perturbations and reconstruct a good quality surface.

Synthetic data : First, images are generated assuming Lambertian reflectance model, distant point source lighting and constant albedo. These images are then corrupted with Gaussian noise to simulate the realistic case. We try to recover the surface normals $\left(n_{x}, n_{y}, n_{z}\right)$. The gradient field is then estimated by $p=-\frac{n_{x}}{n_{z}}$ and $q=-\frac{n_{y}}{n_{z}}$. Results are presented in Figures 4 and 5 and the MSE in 2 As can be seen, the proposed solution performs better thanks to its 


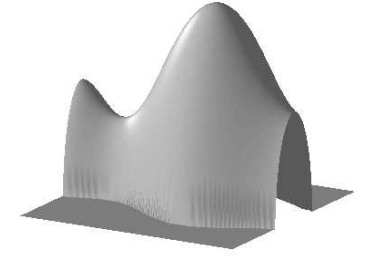

(a) Ground Truth

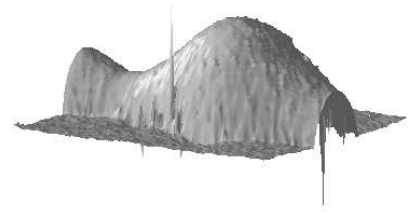

(d) M-estimator [2]

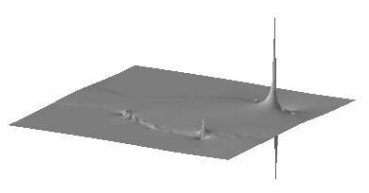

(b) Least Squares [15]

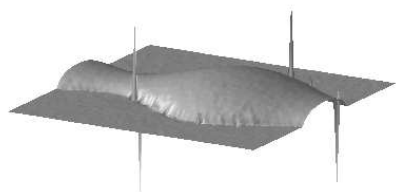

(e) $l_{1}$-minimization [14]

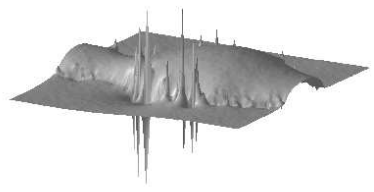

(c) Diffusion [2]

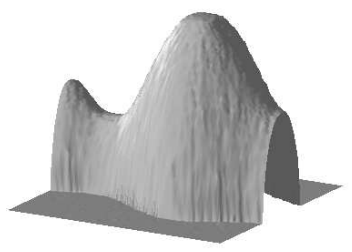

(f) Triple Sparsity

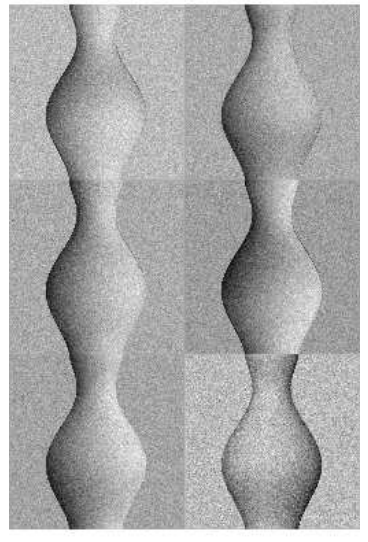

(g) Noisy input images

Figure 5: Photometric stereo on noisy Vase dataset ( $\sigma=14 \%$ of the maximum intensity). The proposed solution performs a better feature preserving reconstruction even in challenging mixed noise/outliers situations (parameters : $\lambda_{1}=0.3, \lambda_{2}=$ $3 \times 10^{-4}, \gamma=0.01, p_{1}=p_{2}=p_{3}=0.1$ ).

ability to handle both noise and outliers in the gradients.

Real data : Calibrated photometric stereo is performed on noisy images using 5 instances of the Octopus ${ }^{1}$ dataset (around $\sigma=2.9 \%$ of the maximum intensity). Results are presented in Figure 6. The MSE is reported in Table 2. The methods Diffusion (c) and M-estimator (d) are able to recover a better surface than the Least Squares method (b), but they produce deformed and noisy surfaces. Although the $l_{1}$-minimization method is marginally less successful compared to Diffusion (c) and M-estimator (d) in terms of the MSE, it better corrects the outliers and produces an improved feature preserving reconstruction. The proposed method (f) corrects even more outliers than the $l_{1}$ minimization method and also smooths simultaneously the surface, resulting in a better preserved surface shape. The produced result with the proposed method is better both visually and in terms of the MSE.

\section{Conclusions}

We propose a new approach to robust surface reconstruction using a triple sparsity prior : a double sparsity to efficiently handle outliers and a third sparsity to deal with noisy gradients and produce a smooth surface. We show the benefits of using a double sparsity prior instead of a single one in terms of robustness to outliers. We show that a third sparsity is important in the case of noisy gradients. We provide extensive experiments, first on near-exact recovery of sparse gradient signals from corrupted gradients, then on surface reconstruction and three Photometric Stereo

${ }^{1}$ The dataset is publicly available on Neil Alldrin's homepage : vision.ucsd.edu/ nalldrin/research/ examples including real data. While the method is computationally more expensive than solving the Poisson equation, it is much more effective in correcting outliers and, at the same time, smooths the surface, producing consistently better feature preserving reconstructions.

\begin{tabular}{|c|c|c|}
\cline { 2 - 3 } \multicolumn{1}{c|}{} & Method & MSE \\
\hline \multirow{4}{*}{ Mozart } & Least Squares & 0.357 \\
\cline { 2 - 3 } & Diffusion & 0.310 \\
\cline { 2 - 3 } & M-estimator & 0.239 \\
\cline { 2 - 3 } & $l_{1}$ & 0.354 \\
\hline \multirow{4}{*}{ Vase } & Triple Sparsity & $\mathbf{0 . 0 2 4}$ \\
\cline { 2 - 3 } & Least Squares & 0.355 \\
\cline { 2 - 3 } & Diffusion & 0.362 \\
\cline { 2 - 3 } & M-estimator & 0.278 \\
\cline { 2 - 3 } & $l_{1}$ & 0.212 \\
\hline \multirow{5}{*}{ Octopus } & Triple Sparsity & $\mathbf{0 . 0 1 0}$ \\
\cline { 2 - 3 } & Least Squares & 0.248 \\
\cline { 2 - 3 } & Diffusion & 0.135 \\
\cline { 2 - 3 } & M-estimator & 0.123 \\
\cline { 2 - 3 } & Triple Sparsity & 0.191 \\
\cline { 2 - 3 } & $l_{1}$ & $\mathbf{0 . 0 9 2}$ \\
\hline
\end{tabular}

Table 2: Normalized MSE of the PS results.

\section{Acknowledgements}

Hicham Badri's PhD is funded by an INRIA (Direction of Research) CORDI-S grant. He is making a PhD in cosupervision with INRIA and Mohammed V-Agdal University - LRIT, Associated Unit to CNRST (URAC 29). We 


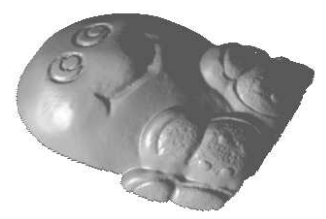

(a) Ground Truth

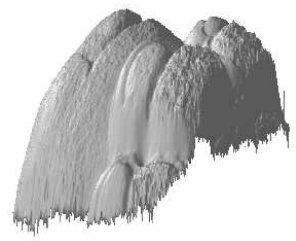

(d) M-estimator [2]

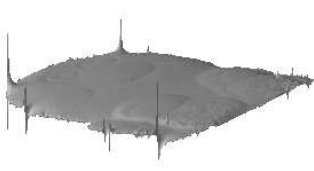

(b) Least Squares [15]

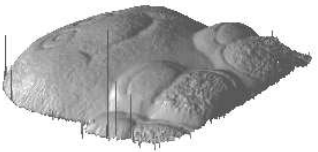

(e) $l_{1}$-minimization [14]

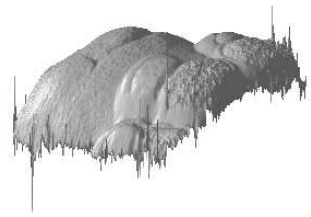

(c) Diffusion [2]

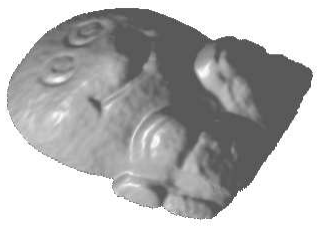

(f) Triple Sparsity

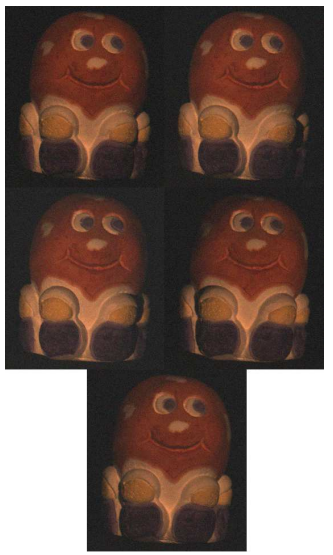

(g) Noisy input images

Figure 6: Photometric stereo on noisy real Octopus dataset. The proposed method (f) is able to recover a good quality surface from very noisy images. The other methods (b), (c), (d) and (e) produce deformed and noisy surfaces (parameters : $\lambda_{1}=0.5$, $\left.\lambda_{2}=2 \times 10^{-5}, \gamma=10^{-4}, p_{1}=p_{2}=0.4, p_{3}=0.8\right)$.

would like to thank Amit Agrawal for kindly providing the Mozart and Vase datasets.

\section{References}

[1] A. Agrawal, R. Chellappa, and R. Raskar. An algebraic approach to surface reconstruction from gradient fields. In ICCV 2005 , Volume 1, pages 174-181. IEEE Computer Society, 2005.

[2] A. Agrawal, R. Raskar, and R. Chellappa. What is the range of surface reconstructions from a gradient field? In Proceedings of the 9th European conference on Computer Vision Volume Part I, ECCV'06, pages 578-591, Berlin, Heidelberg, 2006. Springer-Verlag.

[3] R. Chartrand. Fast algorithms for nonconvex compressive sensing: Mri reconstruction from very few data. In Int. Symp. Biomedical Imaing, 2009.

[4] Z. Du, A. Robles-Kelly, and F. Lu. Robust surface reconstruction from gradient field using the 11 norm. DICTA '07, pages 203-209. IEEE Computer Society, 2007.

[5] R. Fattal, D. Lischinski, and M. Werman. Gradient domain high dynamic range compression. ACM Trans. Graph., 21(3):249-256, July 2002.

[6] R. T. Frankot and R. Chellappa. A method for enforcing integrability in shape from shading algorithms. IEEE Trans. Pattern Anal. Mach. Intell., 10(4):439-451, July 1988.

[7] M. Harker and P. O'Leary. Least squares surface reconstruction from gradients: Direct algebraic methods with spectral, tikhonov, and constrained regularization. In $C V P R$, pages 2529-2536. IEEE, 2011.

[8] B. K. P. Horn. Height and gradient from shading. Int. J. Comput. Vision, 5(1):37-75, Sept. 1990.

[9] P. Kovesi. Shapelets correlated with surface normals produce surfaces. In Proceedings of the Tenth IEEE International Conference on Computer Vision - Volume 2, pages 994-1001. IEEE Computer Society, 2005.
[10] A. Levin, A. Zomet, S. Peleg, and Y. Weiss. Seamless image stitching in the gradient domain. In In Proceedings of the European Conference on Computer Vision, 2006.

[11] V. M. Patel, R. Maleh, A. C. Gilbert, and R. Chellappa. Gradient-based image recovery methods from incomplete fourier measurements. IEEE Transactions on Image Processing, 21(1):94-105, 2012.

[12] P. Pérez, M. Gangnet, and A. Blake. Poisson image editing. In ACM SIGGRAPH 2003 Papers, SIGGRAPH '03, pages 313-318, New York, NY, USA, 2003. ACM.

[13] N. Petrovic, I. Cohen, B. J. Frey, R. Koetter, and T. S. Huang. Enforcing integrability for surface reconstruction algorithms using belief propagation in graphical models. In CVPR (1), pages 743-748. IEEE Computer Society, 2001.

[14] D. Reddy, A. K. Agrawal, and R. Chellappa. Enforcing integrability by error correction using $l_{1}$-minimization. In CVPR, pages 2350-2357, 2009.

[15] T. Simchony, R. Chellappa, and M. Shao. Direct analytical methods for solving poisson equations in computer vision problems. IEEE Trans. Pattern Anal. Mach. Intell., 12(5):435-446, May 1990.

[16] J. Sun, Z. Xu, and H.-Y. Shum. Image super-resolution using gradient profile prior. In CVPR. IEEE Computer Society, 2008.

[17] R. J. Woodham. Shape from shading. chapter Photometric method for determining surface orientation from multiple images, pages 513-531. MIT Press, Cambridge, MA, USA, 1989.

[18] L. Xu, S. Zheng, and J. Jia. Unnatural $l_{0}$ sparse representation for natural image deblurring. In Proceedings of the 2013 IEEE Conference on Computer Vision and Pattern Recognition, CVPR '13, pages 1107-1114, Washington, DC, USA, 2013. IEEE Computer Society. 
Accepted for IEEE Conference on Computer Vision and Pattern Recognition (CVPR) 2014

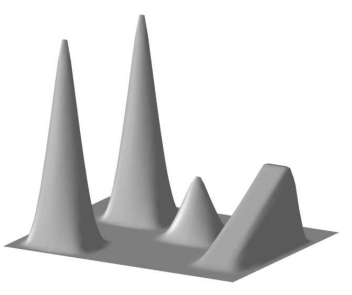

(a) Ground Truth

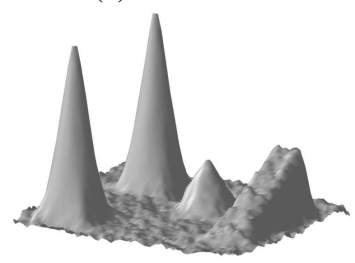

(d) M-estimator [2]

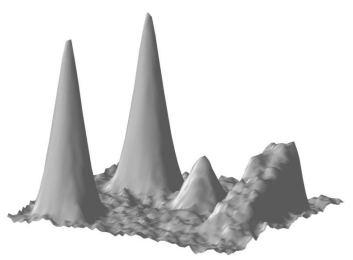

(b) Least Squares [15]

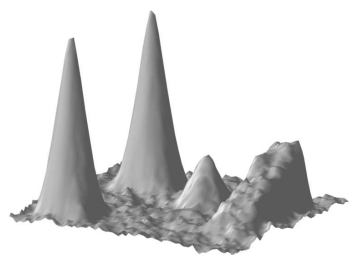

(e) $l_{1}$-minimization [14]

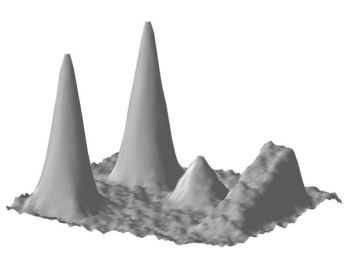

(c) Diffusion [2]

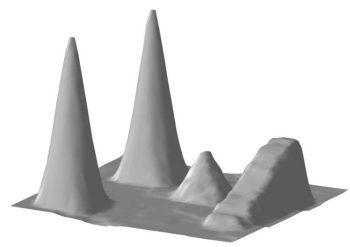

(f) Triple Sparsity

Figure 7: Reconstructed surface when the gradient field is corrupted by Gaussian noise only $(\sigma=10 \%)$.

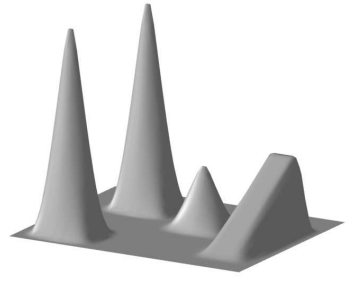

(a) Ground Truth

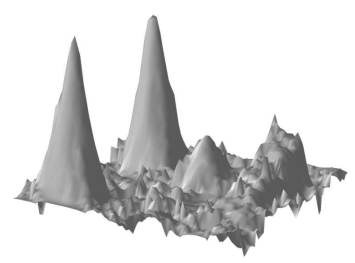

(d) M-estimator [2]

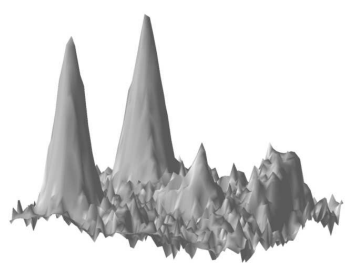

(b) Least Squares [15]

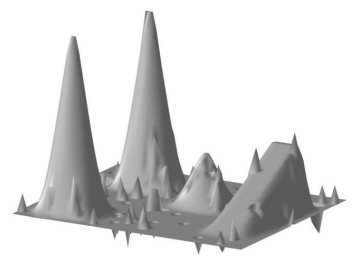

(e) $l_{1}$-minimization [14

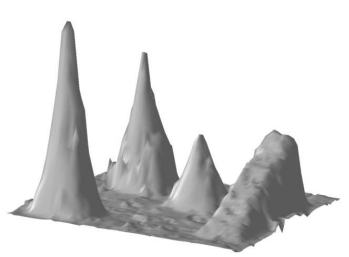

(c) Diffusion [2]

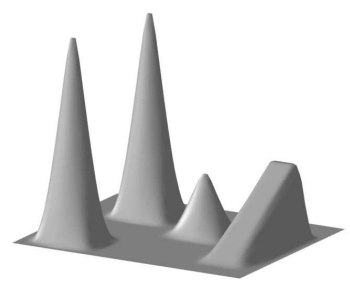

(f) Triple Sparsity

Figure 8: Reconstructed surface when the gradient field is corrupted by outliers only (10\%).

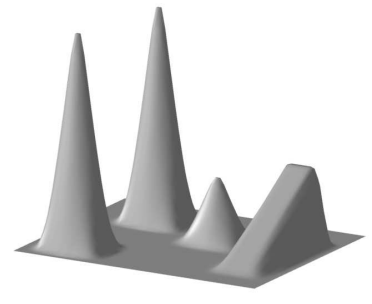

(a) Ground Truth

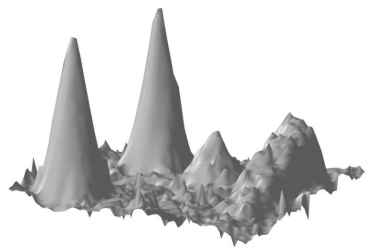

(d) M-estimator [2]

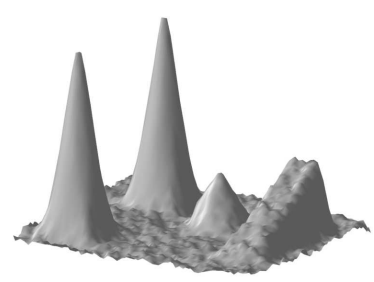

(b) Least Squares [15]

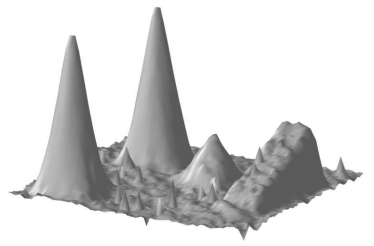

(e) $l_{1}$-minimization [14

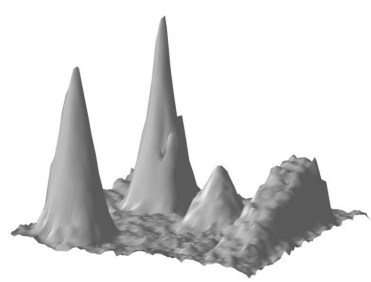

(c) Diffusion [2]

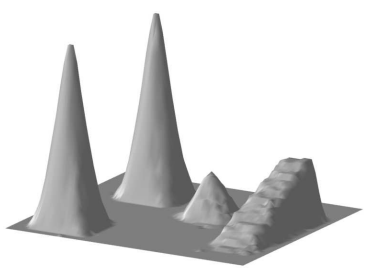

(f) Triple Sparsity

Figure 9: Reconstructed surface when the gradient field is corrupted by outliers $(7 \%)$ and noise $(\sigma=7 \%)$. 